\title{
Preparation of High Coercive-Force Al-Substituted Bi-DyIG Fine Particles and Coating Films
}

\author{
Tae-Youb Kim \\ Teruyoshi Hirano \\ (Tokyo Institute of Technology) \\ Tatsuru Namikawa \\ (Toppan Printing, Co., Ltd.) \\ Yohtaro Yamazaki \\ (Tokyo Institute of Technology) \\ (Tokyo Institute of Technology)
}

In garnet coating films, higher coercive force $H_{\mathrm{C}}$ and lower absorption coefficient $\alpha$ are required to develop a practical magnetooptical recording media. One of the solutions can be found at a magnetic compensation composition of Al-substituted iron garnet particles. Since fine particles of $\mathrm{Bi}-\mathrm{DyAlIG}\left(\mathrm{BiDy}_{2} \mathrm{Al}_{y} \mathrm{Fe}_{5-y} \mathrm{O}_{12}: 0 \leqq y \leqq 1.1\right)$ exhibit a magnetic compensation at room temperature, we investigated the effect of magnetic compensation in Al-substituted Bi-DyIG fine particles.

The Bi-DyAlIG fine particles were prepared by coprecipitation and heat treatment. The heat treatment was carried out at $750^{\circ} \mathrm{C}$ for $4 \mathrm{~h}$. The fine particles were milled with an epoxy binder by a milling machine. The thin films were prepared with a coating technique. Nano-size fine particles of Bi-DyAlIG had been dispersed in the plastic binders on Cornig \#7059 glass sheets.

The fine particle $(y=0.9)$ has a high coercive force of about 450 Oe and only $0.4 \mathrm{emu} / \mathrm{g}$. All the X-ray diffraction peaks of the fine particles were assigned to garnet. The Faraday rotation $\theta_{\mathrm{F}}$ was decreased with increasing $y$. Near $y=0.8$ the rotating direction of the $\theta_{\mathrm{F}}$ was reversed. These results lead to a compensation of the magnetic moments. The size of fine particles in the coating films was about $10 \mathrm{~nm}$ which is much smaller than the wavelengths of the lights utilized in the optical devices.

Key words: Bi-DyAlIG, coprecipitation, magnetic compensation, Faraday rotation, reverses, magnetooptical device.

\section{Introduction}

The increase in the coercive force of magnetic particles and in the lower absorption coefficient have been one of the major objectives for applying the garnet particles to magnetooptical recording media. The fine particles of garnet require more effort to increase the coercive force than the thin films because of their low internal stresses [1][2]. In case the magnetic compensation occurs in Alsubstituted iron garnet particles, the coercive force of the particles will have a huge value and the absorption coefficients of coating films will be improved. At present, $\mathrm{BiDy}_{2} \mathrm{Al}_{y} \mathrm{Fe}_{5-y} \mathrm{O}_{12}$ is one of the candidates, however, the substitution content $y$ for the magnetic compensation of the fine particles is not clear with different reported values of $y=0.35$ [3] and $y=1.2$ [4]. And any attempts to prepare the coating films with the fine particles with these composition have not been reported. Fine particles of the Bi-DyAlIG exhibit a magnetic compensation at room temperature, therefore we investigated the effect of the magnetic compensation in Al-substituted $\mathrm{Bi}$-DyIG fine particles.

In this paper, we precisely determined the magnetic compensation composition of $\mathrm{BiDy}_{2} \mathrm{Al}_{y} \mathrm{Fe}_{5-y} \mathrm{O}_{12}(0 \leqq y \leqq 1.1)$ fine particles, and the structural and magnetooptical properties of the coating films made from the fine particles were also investigated. 


\section{Experimental}

\subsection{Preparation of garnet fine particles}

The Al-substituted Bi-DyIG fine particles were prepared by coprecipitation [5][6] and a heat treatment. Aqueous nitrate solutions of $\mathrm{Bi}$ (Bismuth(III) Nitrate Pentahydrate), Dy (Dysprosium(III) Nitrate Pentahydrate) and $\mathrm{Fe}$ (Iron(III) Nitrate Enneahydrate) were mixed where the ratio of the cations corresponded to the composition of $\mathrm{BiDy}_{2} \mathrm{Al}_{y} \mathrm{Fe}_{5-y} \mathrm{O}_{12}$. Then the aqueous solution of Al nitrate (Aluminium(III) Nitrate Enneahydrate) was added. The concentration of $\mathrm{Al}$ in the samples was from $y=$ 0.0 to 1.1 in the formula unit. Then the solution was mixed with an $\mathrm{NH}_{4} \mathrm{OH}$ solution with stirring at room temperature. The concentration of the alkaline solution was adjusted so that the $p \mathrm{H}$ of the mixed solution was 10.0 at the end of the coprecipitation reaction. The obtained slurry was washed, filtered in aspirator and dried at $120^{\circ} \mathrm{C}$ for $1.5 \mathrm{~h}$. Then the coprecipitates were heated in air at $750^{\circ} \mathrm{C}$ for $4 \mathrm{~h}$ to form fine garnet particles. The crystal phases in the particles were examined by X-ray diffraction (XRD) (RINT2100V; Rigaku) analysis with a $\mathrm{Cu}-\mathrm{K} \alpha$ source. The measurement was made with standard high-purity silicon powder. Saturation magnetization $M_{\mathrm{s}}$ and coercive force $H_{\mathrm{C}}$ of the fine particles were measured with a vibrating sample magnetometer (VSM) (BHV55; Riken Denshi) at room temperature. The contents of the cations in the coprecipitates were analyzed by inductively coupled plasma atomic emission spectrometry (ICP-AES) (SPS4000; Seiko Instruments).

\subsection{Preparation of the coating films}

The fine particles were mixed with an epoxy binder (Epo-tek 396; Epoxytechnology) dissolved in a cyclohexanone and milled with a planetary milling machine (Pulverisette 7; Fritsch) for 30h. They were then coated on Corning \#7059 glass substrates using a rod coater. The coating films were dried at $80^{\circ} \mathrm{C}$ for $2 \mathrm{~h}$ in an oven. The volume content of the fine particles in the coating films was about 0.35 . The coating film thickness was measured with a surface step analyzer (DEKTAK 3030; Sloan) and the coating film surface was observed with a scanning electron microscope (SEM) (S-4000; Hitachi). The thickness of the coating films was about $1.2 \sim 1.7 \mu \mathrm{m}$. It was controlled by the viscosity of the ink and the mesh of the rod coater. The magnetic properties of the coating films were measured with the VSM. The fine particles in the coating films were observed with an atomic force microscope (AFM) (SPI3700; Seiko Instruments). Faraday rotation $\theta_{\mathrm{F}}$ was measured by the polarization modulation method (MOE-7; Jasco). The absorption coefficient $\alpha$ was measured with a spectrophotometer (U2000A; Hitachi). The optical measurements were carried out in the visible wavelength region from $450 \mathrm{~nm}$ to $700 \mathrm{~nm}$.

\section{Results and discussion}

\subsection{Properties of the garnet fine particles}

The X-ray diffraction patterns of the Bi-DyAlIG fine particles are shown in Figure 1. We investigated the X-ray diffraction patterns with the JCPDS data, when the patterns were identified to garnet. The phase of the fine particles from $y$ $=0.0$ to 1.1 is assigned to garnet.

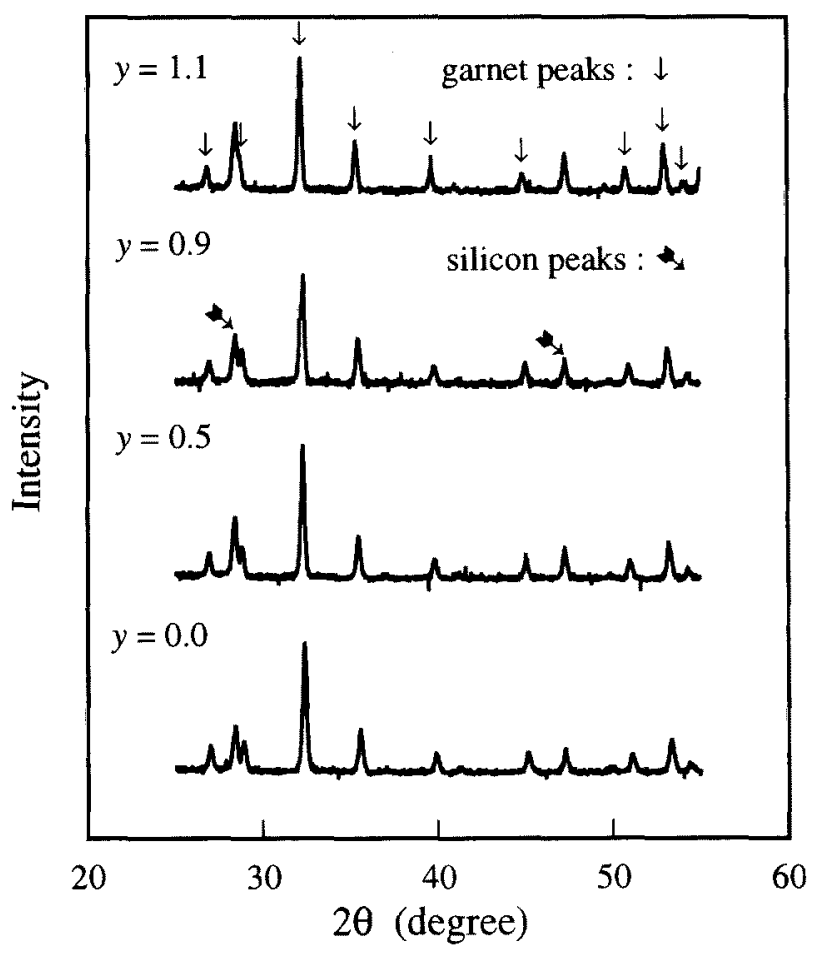

Fig. 1. X-ray diffraction patterns of $\mathrm{BiDy}_{2} \mathrm{Al}_{y} \mathrm{Fe}_{5-y} \mathrm{O}_{12}$ fine particles after heat treatment in $750^{\circ} \mathrm{C}$ for $4 \mathrm{~h}$. 
Figure 2 shows the $M-H$ curves of the Bi-DyAlIG fine particles at room temperature under the magnetic field up to $20 \mathrm{kOe}$. All the particles showed unsaturated magnetization curves. The thickness of magnetic domain walls is about 20 $\mathrm{nm}$ [7]. This value is much larger than that of our observation of about $10 \mathrm{~nm}$ which is described in the following section. The size of about $15 \mathrm{~nm}$ for Bi-YIG particles was also reported [8]. Therefore the size of the particles is much smaller than the thickness of domain walls. We presume that the presence of superparamagnetic particles as a mixture is the reason for the unsaturated $M-H$ curves.

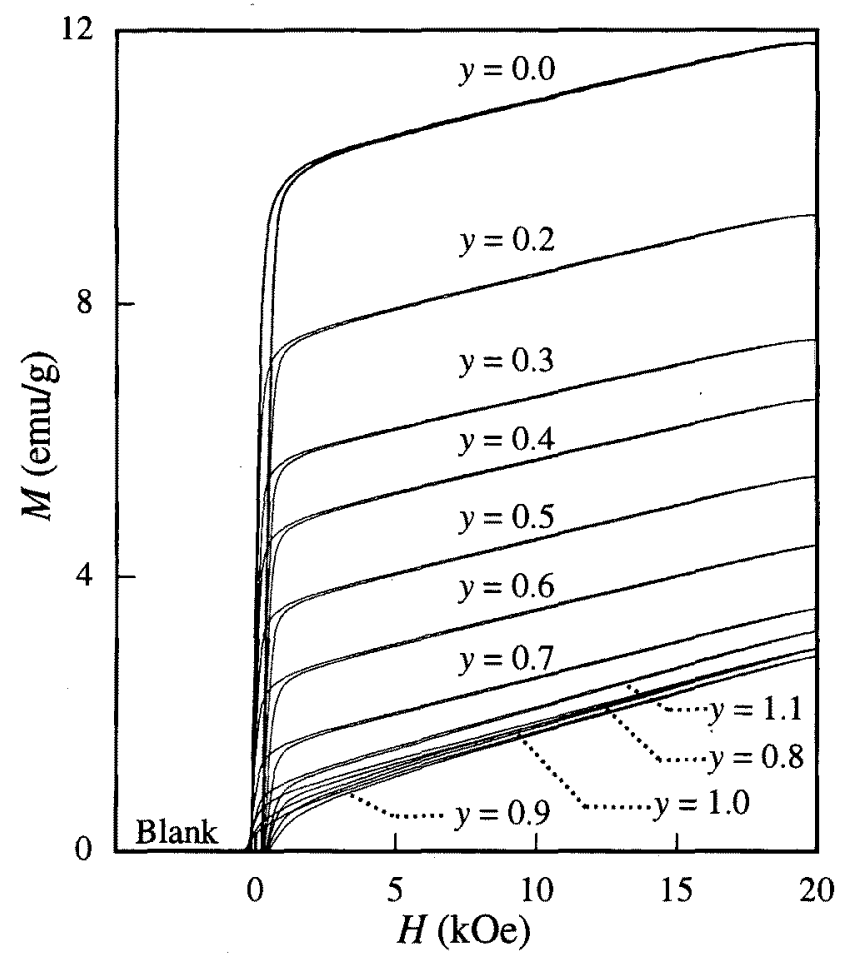

Fig. 2. $M-H$ curves of the $\mathrm{BiDy}_{2} \mathrm{Al}_{y} \mathrm{Fe}_{5-y} \mathrm{O}_{12}$ fine particles at room temperature under the magnetic field up to $20 \mathrm{kOe}$.

Figures 3 and 4 show the magnetization and coercive force $H_{\mathrm{C}}$ of the Bi-DyAlIG fine particles at $H=3 \mathrm{kOe}\left(M_{3 \mathrm{kOc}}\right)$ as a function of $y$. The $M_{3 \mathrm{kOe}}$ decreased with increasing $y$, and became less than $1 \mathrm{emu} / \mathrm{g}$ in the composition range of $0.8<y<$ 1.0 , which is indicative of the magnetic compensation of the Bi-DyAlIG fine particles. We determined the contents of $\mathrm{Al}$ ions occupied on the $16 a$ site $\left(\mathrm{x}_{\mathrm{a}}\right)$ and $24 d$ site $\left(\mathrm{x}_{\mathrm{d}}\right)$ using the saturation magnetizations $M_{\mathrm{S}}$ of bulk DyIG and YIG [9] on the basis of the Neel model. The estimated values of $x_{a}$ and $x_{d}$ are 0.18 and 0.72 ,

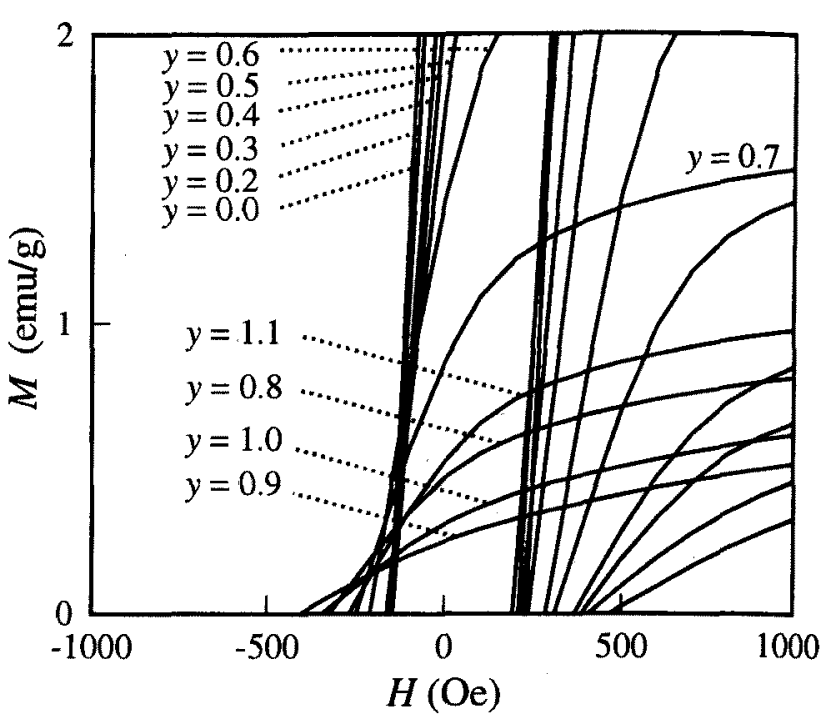

Fig. 3. Hysteresis loops of the $\mathrm{BiDy}_{2} \mathrm{Al}_{y} \mathrm{Fe}_{5-y} \mathrm{O}_{12}$ fine particles.

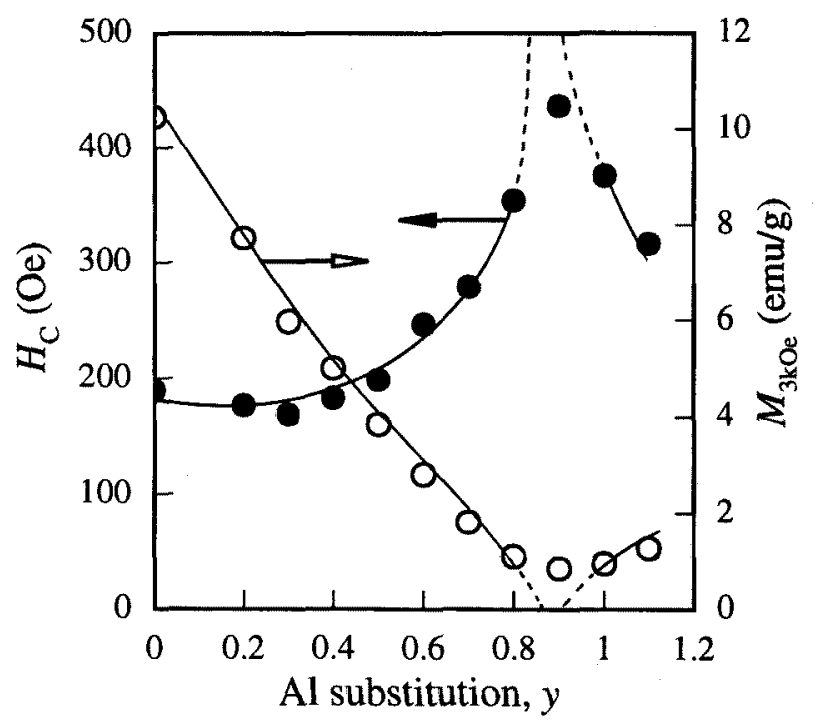

Fig. 4. Magnetization at $H=3 \mathrm{kOe}\left(M_{3 \mathrm{kOe}}\right)$ and coercive force $\left(H_{\mathrm{C}}\right)$ of $\mathrm{BiDy}_{2} \mathrm{Al}_{y} \mathrm{Fe}_{5-y} \mathrm{O}_{12}$ fine particles as a function of $\mathrm{Al}$ substitution.

respectively. Moreover, the $H_{\mathrm{c}}$ of the fine particles was increased at this composition. The fine particles with $y=0.9$ has a high coercive force of about 450 Oe which is a factor of 3 larger than the previously reported value [10]. We should shortly comment on the magnetic compensation in Bi-DyAlIG fine particles. There are some reports showing the different compensation compositions, e.g., $y=1.2$ [4] and $y=0.35$ [3]. We reliably attribute this discrepancy to the different contents of the $\mathrm{Al}$ atoms on each site. However, the magnetic compensation composition 
of $y=0.35$ cannot be explained in terms of the saturation magnetizations of the bulk. This requires novel spin structure in the particle systems. It was previously reported that the saturation magnetization reduced by a factor of 2 due to the spin canting configuration in the surface layers of the Bi-DyAlIG fine particles [3][11]. Thus the spin structure characteristic of the particles also should be taken into account as we determine the magnetic compensation composition, although we cannot demonstrate the spin structure of the present Bi-DyAlIG particles.
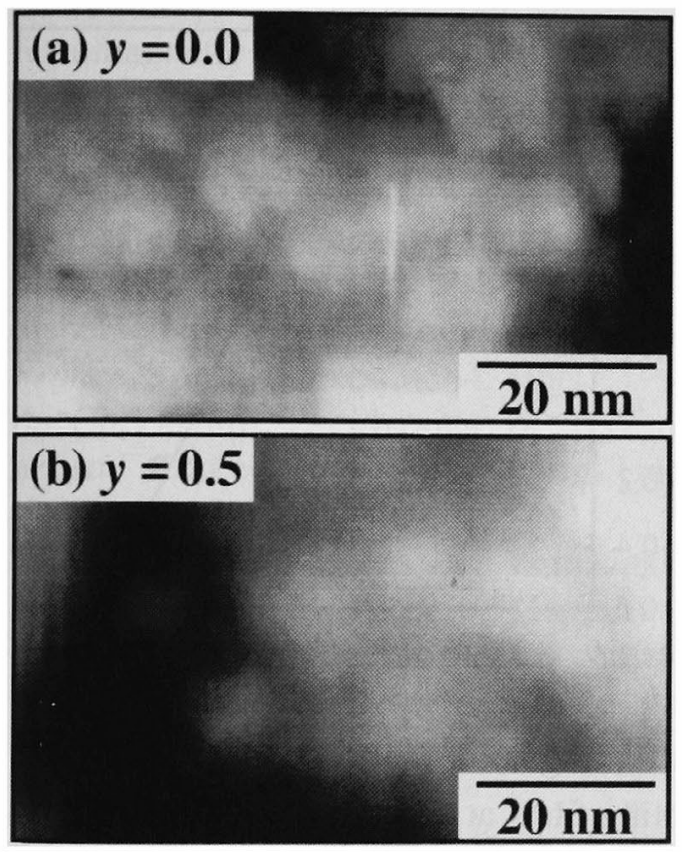

(c) $y=0.9$
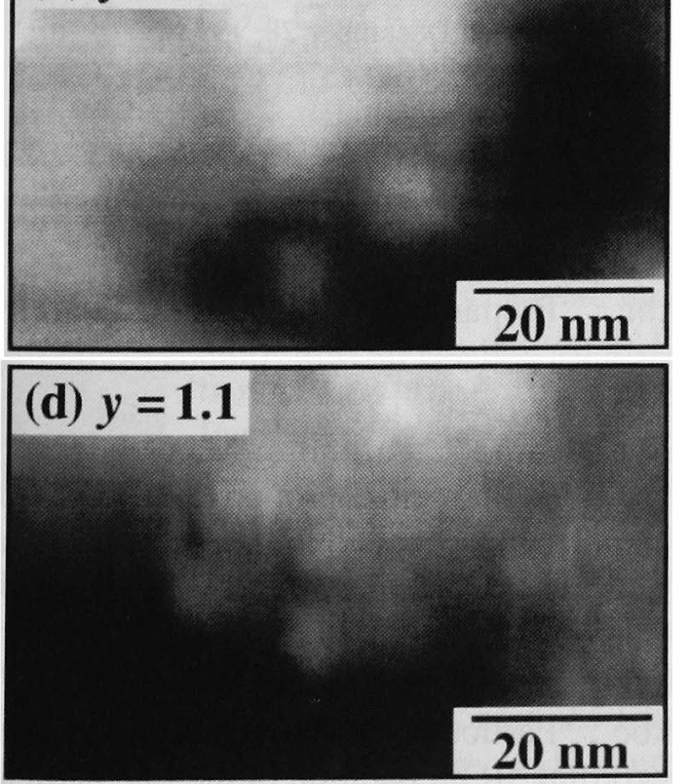

Fig. 5. AFM images of $\mathrm{BiDy}_{2} \mathrm{Al}_{y} \mathrm{Fe}_{5-y} \mathrm{O}_{12}$ fine particles in the coating films.

\subsection{Properties of the coating films}

Figures 5 (a), (b), (c) and (d) show AFM images of the Bi-DyAlIG fine particles in the coating films which were milled for $30 \mathrm{~h}$. The primary size of the fine particles is about $10 \mathrm{~nm}$. The size is almost the same as that of the coprecipitated fine particles. From the SEM images of the coating films, no aggregate of the fine particles was observed. It is confirmed that the fine particles are uniformly dispersed. The size is much smaller than the wavelengths of the read and write lasers for visible light and near future magnetooptical storage system.
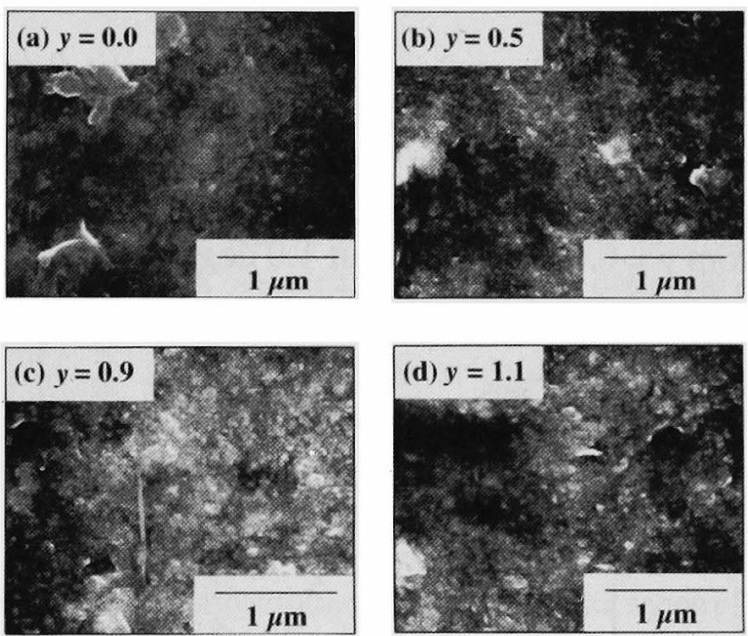

Fig. 6. SEM images of $\mathrm{BiDy}_{2} \mathrm{Al}_{y} \mathrm{Fe}_{5-y} \mathrm{O}_{12}$ fine particlesin the coating films.

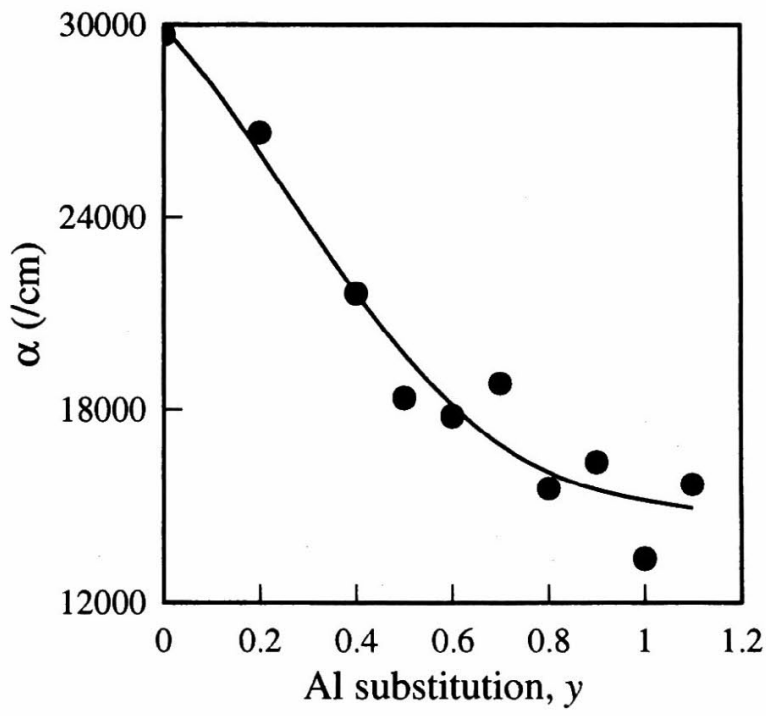

Fig. 7. Absorption coefficient $\alpha$ of $\mathrm{BiDy}_{2} \mathrm{Al}_{y} \mathrm{Fe}_{5-y} \mathrm{O}_{12}$ coating films at $520 \mathrm{~nm}$. 


\subsection{Magnetooptical properties of the coating films}

Figure 7 shows the absorption coefficient $\alpha$ of the Bi-DyAlIG coating films at wavelength 520 $\mathrm{nm}$. The absorption coefficient at the coating films was decreased with increasing $y$.

Figure 8 shows the Faraday rotation $\theta_{\mathrm{F}}$ of the Bi-DyAlIG coating films at the $520 \mathrm{~nm}$, where anticlockwise rotation of the polarization was defined to be positive of $\theta_{\mathrm{F}}$ when viewed along the light propagation. The $\theta_{\mathrm{F}}$ decreases with increasing $y$. In the region from $y=0.8$ to 0.9 , direction of the Faraday rotation was reversed. This result leads to a compensation of the magnetic moments.

The relation between the figure of merit $\theta_{\mathrm{F}} / \alpha$ at $520 \mathrm{~nm}$ of the coating films and $y$ are shown in figure 9. The $\theta_{\mathrm{F}} / \alpha$ for the coating films shows the maximum value at $y=0.4$. The value of $\theta_{\mathrm{F}} / \alpha$ at from $y=0.0$ to 0.6 is about 1.0 degree.

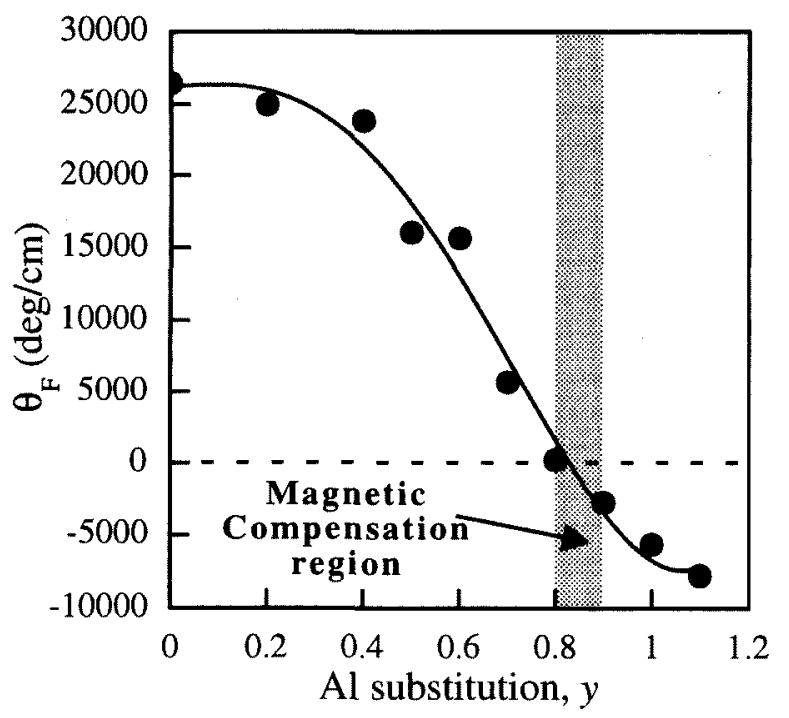

Fig. 8. Faraday rotation $\theta_{\mathrm{F}}$ of $\mathrm{BiDy}_{2} \mathrm{Al}_{y} \mathrm{Fe}_{5-y} \mathrm{O}_{12}$ coating films at $520 \mathrm{~nm}$.

\section{Conclusions}

We successfully synthesized nano-size Alsubstituted Bi-DyIG $\left(\mathrm{BiDy}_{2} \mathrm{Al}_{y} \mathrm{Fe}_{5-y} \mathrm{O}_{12} ; 0 \leqq y \leqq\right.$ 1.1) fine particles by a coprecipitation and heat treatment. Thin films were prepared by coating an ink which was made through a planetary process milling. The magnetic and magnetooptical properties of the fine particles and coating films were investigated.
The fine particles of $\mathrm{BiDy}_{2} \mathrm{Al}_{y} \mathrm{Fe}_{5-y} \mathrm{O}_{12}$ exhibited a magnetic compensation at room temperature with the composition of $y=0.9$. Fine particles of $y=$ 0.9 have a high coercive force of $450 \mathrm{Oe}$. The Faraday rotation $\theta_{\mathrm{F}}$ decreased with increasing $y$. In the region from $y=0.8$ to 0.9 , the rotating direction of the $\theta_{\mathrm{F}}$ was reversed. The figure of merit $\theta_{\mathrm{f}} / \alpha$ of the coating films from $y=0.0$ to 0.6 is about 1.0 degree at $520 \mathrm{~nm}$. These results indicate that the coating films are promising candidate for a new large scale magnetooptical storage media.

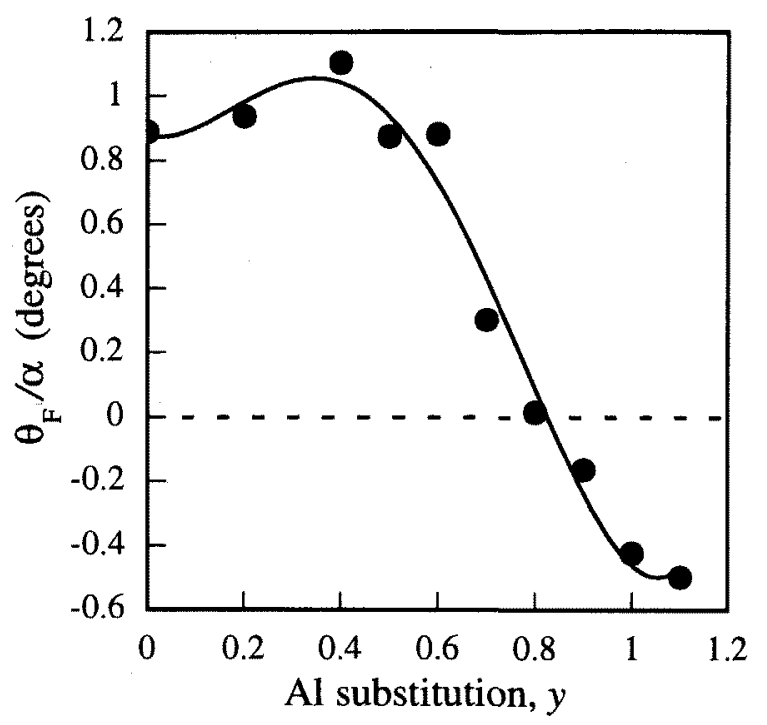

Fig. 9. Figure of merit of $\mathrm{BiDy}_{2} \mathrm{Al}_{y} \mathrm{Fe}_{5-y} \mathrm{O}_{12}$ coating films at $520 \mathrm{~nm}$.

(Manuscript received June 26, 1998, revised December 28, 1998)

\section{REFERENCES}

1) T. Fujimoto, Y. Kumura, M. Gomi and $M$. Abe : "Preparation of garnet structure fine particles" Digest of 14th Annual Conf. Magn. Soc. Jpn., p.209, 1990.

2) Y. Kumura, T. Fujimoto, M. Gomi and M. Abe : "Fundamental properties of particulate media for magneto-optical recording II and III" Digest of 14th Annual Conf. Magn. Soc. Jpn., pp.210-211, 1990.

3) T. Fujimoto, Y. Kumura, M. Gomi and M. Abe : "Particulate films for magneto-optical recording III preparation of the fine particles", J. Magn. Soc. Jpn., vol. 15,Sppl.S1, pp.263265, 1991. 
4) P. Hansen and J. -P. Krumme : "Magnetic and magneto-optical of garnet films", Thin Solid Films, vol 114, pp.69-107, 1984.

5) T. Hirano, T. Namikawa and Y. Yamazaki : "Bi-YIG magneto-optical coated films for visual applications", IEEE Trans. Magn., vol 31(6), pp.3280-3282, 1995.

6) E. Komuro, T. Hirano, N. Kawai, T. Namikawa and Y. Yamazaki : "Preparation of magneto-optical films with Bi-YIG particles", J. Magn. Soc. Jpn., vol 19, Sppl.S1, p.189, 1995.

7) C. Kittel : "Theory of the structure of ferromagnetic domains in films and small particles", Phys. Rev., vol 70, pp.965-971, 1946.

8) Y. Yamazaki, T. Namikawa, T. Hirano and K. Yoshida : " Magnetic and magneto-optical properties of nano-size Bi-YIG particles dispersed in a plastic binder", J.Phys IV France, SpplC1, pp.543-544, 1997.

9) S. Chikazumi : "Physics of ferromagnetism", 2nd ed. New York, Oxford University Press, 1997.

10) T. Hirano, K. Yoshida, Y. Yamazaki, T. Namikawa and M. Kishi : "Magnetic and magneto-optical properties of Co-containing Bi-DyIG particles dispersed in a plastic binder", IEEE Trans. Magn., vol. 33, pp.3277-3279, 1997.

11) K. Haneda : "Recent advances in the magnetism of fine particles", Can. J. Phys., vol. 65, pp.1233-1244, 1987.

Kim, Tae - Youb was born in Pusan,

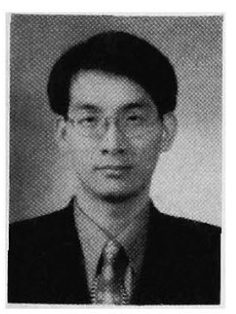
Korea in 1969. He received the B.Sc. degree in Chemistry from Kosin University, Korea, in 1995 and the M.Eng. degree in Electronic Chemistry from Tokyo Institute of Technology, Japan, in 1998. He is currently working toward the Ph.D. degree at Department of Innovative and Engineered Materials in Tokyo Institute of Technology, Japan. He is a member of the Magnetics Society of Japan, the Japan Society of Applied Physics, and the Electrochemical Society of Japan. His research interests are oxide magnetic materials, superparamagnetic garnet particles, and magnetooptical device.
Teruyoshi Hirano was born in Saitama, Japan, on April 5, 1962. He received the M.Sc. degree from Science University of Tokyo in 1987. He received the Dr. Engineering degree from Tokyo Institute of Technology in 1997. He has been with Toppan Printing Co., Ltd. since 1987. His current research interests are the nano size particles dispersed materials and its applications. He is a member of the Magnetics Society of Japan, the Japan Society of Applied Physics, the Electrochemical Society of Japan, and the Material Research Society.

Tatsuru Namikawa was born in Tokyo,

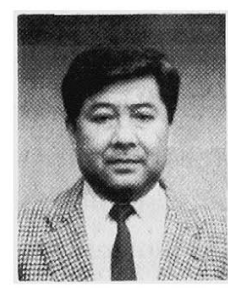
Japan, on February 11, 1939. He graduated from Tokai University in 1964. He received the D.E. degree from Tokyo Institute of Technology in 1983. Since 1964 he has been with the Department of Electronic Chemistry of Tokyo Institute of Technology, Nagatsuta, where he is presently a Associate Professor in the Department of Innovative and Engineered Materials. Dr. Namikawa is a member of the Magnetics Society of Japan, the Japan Society of Powder and Powder Metallurgy, and the Chemical Society of Japan.

Yohtaro Yamazaki was born in Tokyo in

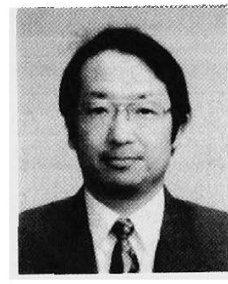
1945. He received the B.Sc. and Ph.D. degrees from Tokyo Institute of Technology in 1969 and 1974 , respectively. From 1981 to 1997 he was an associate professor of Tokyo Institute of Technology. Since 1997 he has been a professor of The Interdisciplinary Graduate School of Science and Engineering, Tokyo Institute of Technology. 1997-99 Dept. Chairman. $\mathrm{He}$ is currently a member of the board of directors of the Magnetics Society of Japan, and the Japan Society of Powder and Powder Metallurgy. He has been active in oxide magnetic materials, and electrochemical energy conversion systems. 